\title{
A Proposal for an Enhanced Inter-Cell Interference Coordination Scheme with Cell Range Expansion in LTE-A Heterogeneous Networks
}

\author{
A. Daeinabi, K. Sandrasegaran \\ Centre for Real-time Information Networks, Faculty of Engineering and Information Technology, \\ University of Technology Sydney, Australia
}

\begin{abstract}
In heterogeneous network, the interference situations may be changed in different locations because of nodes with different powers as well as traffic loads. The Inter-Cell Interference (ICI) problem can occur for both data and control channels. To mitigate ICI problem, enhanced Inter-Cell Interference Coordination (eICIC) techniques are proposed in the Long Term Evolution-Advanced (LTE-A) networks particularly picocells. Moreover, the coverage areas of picocell are usually narrow because of difference between transmission power of macro eNodeB (eNB) and pico eNB. Therefore, traffic load is distributed unequally which can lead to macrocells overloading. To address the coverage problems resulted by nodes with different powers, Cell Range expansion (CRE) technique has been recently proposed. In this paper, the CRE approach is combined with eICIC-time domain scheme to improve system performance using features of both two techniques.
\end{abstract}

Keywords- LTE-Advanced, HetNet, picocell, cell range expansion, enhanced intercell interference coordination.

\section{INTRODUCTION}

In recent years, the demand for mobile broadband services with higher data rates and various types of Quality of Service (QoS) is growing rapidly. This demand has motivated 3GPP to work on Long Term Evolution (LTE) to achieve the peak data rates more than $100 \mathrm{Mb} / \mathrm{s}$, a radionetwork delay of less than $5 \mathrm{~ms}$, the enhanced spectrum efficiency and the bandwidth flexibility. Although LTE Release 8 has many of the features taken into account for future fourth generation (4G) systems [1], the performance of LTE Release 8 does not meet IMT-Advanced requirements defined by the International Telecommunications Union (ITU) for the 4G evolution [2]; therefore other releases have been introduced. The evolved versions (LTE Release 10 and beyond), called LTEAdvanced, can meet the requirements defined by IMTAdvanced (e.g., downlink data rates up to $1 \mathrm{Gbps}$ ) [3].

Since the radio bandwidth is one of the scarce resources in wireless networks, new resource allocation algorithms should be introduced to overcome radio resource limitation particularly when applications with high data rate are deployed. For this purpose, the frequency reuse technique is applied to cellular networks to increase the system capacity. However, the system performance is severely degraded because the interference increases when system uses the frequency reuse factor one. In frequency reuse one all cells use the same frequency band. Intercell interference (ICI) resulted by using the same frequency in neighbouring cells can restrict the $4 \mathrm{G}$ performance in terms of throughput and spectral efficiency. Therefore, the ICI mitigation is a critical point to improve the performance of LTE and LTE-A networks.

In this paper, we study ICI challenges in LTE-A particularly picocells. Picocells are one of the important solutions among low power nodes because they can be efficiently deployed in local regions with high volume traffic (such as hotspots) and improve the overall system capacity. Therefore, the important roles of macrocell and picocell in 4G network motivated us to study the ICI problem between macrocell-picocell and then proposed a new eICIC scheme to improve the system performance.

The rest of the paper is organized as follows. The heterogeneous architecture will be reviewed in Section II. The challenges of macrocell-picocell scenario are underlined in Section III. Section IV introduces the Cell Range Expansion technique. A comprehensive survey of picocell eICIC-time domain schemes is depicted in Section V. Section VI proposed a new eICIC scheme. The conclusion is given in the final Section.

\section{HETEROGENEOUS ARCHITECTURE}

Since data traffic demand in cellular networks is growing exponentially, further improvements in 4G spectral efficiency could be possible by increase of the node densities. However, with current dense deployments, the cell dividing gains are considerably decreased because of severe ICI. Moreover, site acquisition costs can get prohibitively expensive particularly in a space limited dense in urban region [4]. One solution to overcome this issue is the utilization of heterogeneous network (HetNet).

A HetNet consists of macrocells as well as low power nodes (i.e., femto, pico, relay nodes) which can be classified in terms of their transmission powers, antenna heights, the type of access mode provided for UEs, and the backhaul connection to other cells [5], [6]. The goal of using low power nodes is to offload the traffic from the macrocells, enhance coverage and throughput, and increase the spectral efficiency by spatial reuse of spectrum. Picocell is one of the important solutions which can be deployed efficiently in local regions with high volume of traffic (such as hotspots) 
and improves the overall system capacity [7]. Picocells are used with lower transmission power rather than macrocells (in a range from 23 to $30 \mathrm{dBm}$ ) [8, 9]. Since picocells usually work in open access mode then all users can access them. In general, the open access mode means that any user in the network can automatically connect to the hotspots. Picocells are used to improve capacity as well as the coverage of outdoor or indoor regions in environments with inadequate macro penetration. Moreover, the communication between macrocells and picocells is done over the X2 interface.

\section{CHALLENGES OF MACROCELL-PICOCELL SCENARIO}

Using nodes with different transmission powers comes up new challenges in HetNets. In this section, we will describe two important cases of these challenges in macrocellpicocell scenario.

\section{A. Intercell Interference Problem}

When a UE moves away from the serving eNB and becomes closer to other eNBs, the strength of desired received signal decreases and the ICI increases. The impact of ICI in LTE downlink can be analysed according to the received signal to interference and noise ratio (SINR) of $\mathrm{UEm}$ on RBn. Three important factors have a great influence on the SINR of each UE including: (a) Channel gain from eNB to UE, (b)Transmission power of each RB, and (c) RB allocation scheme.

Because of the larger transmission power of the macro eNB, the handover boundary becomes closer to the pico eNB which causes uplink interference challenges. When a UE closed to picocell is served by macro eNB, the strong interference will occur for UE located in the picocells. Consequently, picocells may become underutilized because of the large impacted interference. Moreover, it is clear that a mechanism is needed to allow flexible connection between a serving eNB and a UE and mitigate downlink and uplink interference beneficially [4]. Therefore, one of the important aspects of HetNets is interference management. The interference management should be able to support sufficiently the co-channel deployment of various traffic loads as well as using of different numbers of low power nodes at different geographical regions. In order to coordinate between macro eNB and pico eNB, some messages should be exchanged among them through X2 interface [10].

\section{B. Imbalanced Coverage}

Using nodes with different powers can lead to other challenges such as imbalanced coverage between uplink and downlink. The downlink coverage of the macro eNB is much larger than the coverage of the pico eNBs because of difference in their transmission powers. However, the difference in the transmission power of macrocell and picocells does not affect the coverage in the uplink because in this case the UE is a transmitter and the transmission powers of all UEs are same. Therefore, the eNB that provides the best downlink coverage might be different from the eNB providing best uplink coverage.

\section{CELL RANGE EXPANSION}

In the traditional cell selection method, UEs can select the serving cell by comparing a downlink signal from macro eNBs and pico eNBs called Reference Signal Received Power (RSRP). Then, the cell with higher RSRP is selected as a serving cell. This technique is called max RSRP. However, in HetNets, cell selection based on the strongest downlink RSRP is not the best strategy because UEs connect to a higher power node instead of the lower power nodes at the shortest pathloss distance. Therefore, traffic load is distributed unequally which can lead to macrocells overloading. Moreover, the maximum RSRP method causes a sever uplink interference between macro UEs and pico UEs [11]. Consequently, to address the problems resulted by nodes with different power, new cell selection techniques are required so that the UE connects to cells with a weaker RSRP.

One solution is that an offset value is added to RSRP received from pico eNB so that the UE preferentially selects a pico eNB as the serving cell even when it is not the strongest cell. This technique is known as Cell Range Expansion (CRE). Note that the range expanded area is an area around picocells where UEs connect to pico eNBs because of receiving RSRP plus an offset value. Different offset value ranged from 0 to $20 \mathrm{~dB}$ can be considered and for the offset value equals to 0 , CRE acts as the maximum RSRP technique. However, if the offset value is not selected sufficiently for CRE, the enhancement of network performance cannot compensate the cost of using picocells. Consequently, the offset value should be determined precisely to achieve a good system performance.

Although the CRE significantly mitigates interference in the uplink, the downlink signal quality of UEs located in the range expanded area decreases. Such UEs may suffer from downlink SINRs below $0 \mathrm{~dB}$ because they are connected to cells that do not have the best downlink RSRP [11]. Therefore, in the range expanded area, both data and control channels will suffer from ICI because they are not planned for too low SINR. Consequently, ICI mitigation methods should be deployed for control and data channel in CRE approach to keep the cell coverage and improve the system performance.

\section{ENHANCED INTERCELL INTERFERENCE COORDINATION ALGORITHMS FOR PICOCELL IN LTE-A}

In HetNet, the interference situations may be changed in different locations because of different powers as well as traffic loads. The ICI problem can occur for both data and control channels in range expanded areas. Although in 3GPP, the interference of data channel can be mitigated through ICI mitigation techniques proposed for LTE Release 8, the suggested ICI mitigation methods did not consider the problem of control channel interference. Therefore, in order to keep the cell coverage and improve the system performance, ICI mitigation should be deployed for control and data channel in CRE approach. Moreover, due to small radius of picocells, the LTE ICI mitigation 
schemes such as the fractional frequency reuse based scheme cannot be used. Therefore, to overcome the ICI problem in LTE-A, enhanced Inter-Cell Interference Coordination (eICIC) techniques have recently been proposed and can be divided into three major categories [12]: Time domain, Frequency domain and Power domain. Since the focus of this paper is time-domain, therefore a number of time domain-eICIC techniques proposed for macrocell-picocell configuration are reviewed as follows.

If interference coordination is not deployed for UEs located in range expanded area, they will experience large downlink interference from the macro eNB. The interference problem can be mitigated in time domain through subframe utilization. This utilization is PERFORMED across different cells through protected subframes (PSF) including almost blank subframes (ABS) and reduced power subframes (RPS). ABSs are subframes without any activity [13] or only transmitting the reference signals from macro eNB without any types of control or data signals while in RPS the macro eNBs only decrease the transmission power on the specified subframes. Macrocellpicocell configuration use ABSs at the macrocell and schedule UEs located in the range expanded area within subframes that overlap with the ABSs of the macrocell as shown in figure 1. ABS configuration illustrates how picocells are informed about the interference pattern from the macro eNB and then schedule their UEs on protected subframes to avoid from high power interference. Although the large number of ABSs can reduce the interference and improve the performance of picocell, it will decrease the available transmission time for macrocell. Therefore, the number of ABSs should be selected properly to improve the whole system performance.

Reference [7] investigated the performance of eICIC and CRE in picocell downlink system. In this algorithm, only the macrocell does not transmit on some subframes (protected subframes) for the specific time to protect UEs connected to the picocells because the interference caused by the picocells is not important in a HetNet. Then macrocell uses other resources (non-protected subframes) to send data to its UEs. The ratio of the protected subframes is obtained based on the number of the UEs connected to the picocells without $\mathrm{CRE}$, and the number of the UEs connected to the picocells with CRE. Reference [14] has considered a non-static eICIC scheme using Lightly Loaded $\mathrm{CCH}$ transmission Subframe (LLCS). To mitigate the Physical Downlink Control Channels (PDCCH) interference impacted from the macrocell to the pico CEUs, the proposed algorithm deploys both LLCS and ABS just to the macrocells. Since PDCCH is distributed according to the predetermined hopping pattern over the $\mathrm{CCH}$ area, the interference of PDCCH in neighbour cells can be mitigated by the LLCS.

Reference [15] proposed a method to find the optimal amount of ABS in ABS configuration. This algorithm has assumed that pico UEs are categorized into victim UEs and normal UEs. Moreover, macro eNB works only in non-ABS but pico eNB performs in all subframes and victim UEs are scheduled in ABSs. The optimal selection for victim UEs

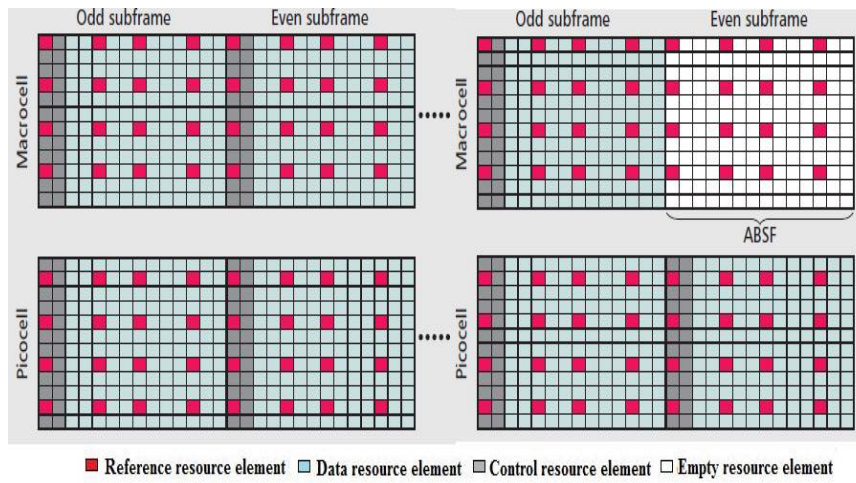

(a)

(b)

Figure 1: ABSs configuration deployed in time domain eICIC for 3GPP heterogeneous networks:

a) Macrocell and picocell subframes without any eICIC, b) Macrocell and picocell subframes with eICIC

and normal UEs is done through the utility function. In [16], two interacting factors have been considered to improve the performance of macrocells and picocells: UE partition and the number of ABSs. A Nash Bargaining Solution [17], [18] has been used to model the UE partition problem. The ABS pattern will be generated based on the obtained subframe ratio and then broadcasted to all eNBs. Reference [19] investigated the effect of static and dynamic protected subframes (PSF) configuration on system performance. A dynamic scheme is used to determine the PSF density (i.e., number of PSF). Therefore, a new metric has been deployed (based on average logarithmic throughput of the macrocell and picocell UEs) to find the suitable PSF density because the low PSF density decreases the performance of pico UEs.

Channel Quality Indicator (CQI) adjustment is another problem which should be taken into account when ABSs are used. Due to the time delay in CQI reporting, it is possible the CQI measured for one type of subframes is deployed for another type of subframes. Therefore, the channel cannot be appropriately estimated and then a wrong modulation and Coding Scheme (MCS) will be selected. Consequently, using incorrect CQI leads to transmission failure or transmission inefficiency. References [20] and [21] worked on CQI adjustment to improve the UE performance. For instance, in [20] the multiple CQI feedback has been used along with the joint decision method. For this purpose, a set of $K$ neighbouring macro and pico eNBs cooperate to select the status of each other.

As a conclusion for the time domain schemes, the interference is mitigated when victim UEs are scheduled on the protected subframes and non protected subframes. When the CRE offset value is low, a small number of UEs connects to the picocells. Consequently, the cell edge throughput becomes higher when the ratio of the subframes allocated to picocell is low since many UEs connected to the macrocell can use more resources. However, when the high CRE offset value is used, a large number of UEs are offloaded and then connect to the picocells. Therefore, the high ratio of the picocells' subframes is required to improve 
the cell edge throughput. On the other hand, the cell throughput decreases by increasing the CRE offset value because the picocell throughput decreases when the UEs far from the picocells are connected to the picocells. Consequently, in time domain eICIC techniques, the ratio of subframes selected for the macrocell-picocell configuration is very crucial factor and an inappropriate ratio can sacrifice the cell edge throughput or cell throughput. Consequently, approaches with a dynamic subframe ratio selection have better performance among proposed time domain eICIC techniques.

\section{THE PROPOSED SCHEME}

As discussed in previous sections, although the CRE can mitigate interference in uplink, it can lead to interference in downlink for UEs located in cell range expanded area (RE $\mathrm{UE}$ ). Especially if the offset value is not selected efficiency, the system performance is degraded in case of using CRE. However, if the CRE technique is combined with one time domain eICIC scheme, it can improve the system performance. This is because when the number of UEs located in cell expanded area can be matched with the number of ABSs, the interference between RE UE and macro UE can be mitigated.

For this purpose, we propose a technique which combines CRE with ABSs scheme. In this scheme, the offset value and ABS ratio can be determined simultaneously based on some metrics such as the throughputs and outage. These metrics are the input of the system and they will be calculated every Time Transmission Interval (e.g., $1 \mathrm{~ms}$ ). The cell monitors the inputs and then makes decision about the next value for offset value and ABS ratio. To find the optimal value for ABS ratio and offset value, an optimization technique is needed.

Another feature of the proposed scheme is that each macro cell can execute it independently based on its performance.

Assessment is based on the MATLAB system level simulation in a LTE downlink network with 19 cells. The network topology is composed of a set of cells and network nodes including macro eNBs, pico eNBs and UEs which are distributed within cells. A certain area is defined for simulation where the eNBs and UEs are located and only in this region, UE movement and transmission are simulated. Each network node is identified by a unique ID and its position is defined using Cartesian system. A wraparound function should be used to make sure that the users do not exit from the simulation area. The simulation length is measured by TTIs which equals $1 \mathrm{~ms}$.

\section{CONCLUSION}

To overcome the ICI problem in macrocell-picocell, several eICIC techniques have recently been proposed for time domain. Approaches with a dynamic subframe ratio selection have better performance among proposed time domain eICIC techniques. To enhance the performance of system, we proposed a scheme combining CRE and ABSs scheme. By this scheme, the offset value and ABS ratio can be selected simultaneously based on system throughputs which can lead to improve the system performance.

\section{REFERENCES}

[1] H.Holma, and A.Toskala, "LTE for UMTS, OFDMA and SC-FDMA Based Radio Access”, Wiley, 2009.

[2] D. Astely, E. Dahlman, A. Furuskar, Y. Jading, M. Lindstrom, and S. Parkvall, " LTE: the evolution of mobile broadband," IEEE Communications Magazine, 2009, vol. 47, no.4, pp.44-51.

[3] 3GPP, TR 36.913, "Requirements for further advancements for Evolved Universal Terrestrial Radio Access (E-UTRA) (LTE-Advanced), Technical Report,” Dec. 2009.

[4] A. Damnjanovic, J. Montojo, W. Yongbin, J. Tingfang, L. Tao, M. Vajapeyam and D. Malladi, “ A survey on 3GPP heterogeneous networks,” IEEE Wireless Communications, Feb.2011, vol.18, no.3, pp.10-21.

[5] 3GPP R1 084026, "LTE-Advanced Evaluation Methodology," Oct. 2008.

[6] S.Brueck, "Heterogeneous networks in LTE-Advanced," $8^{\text {th }}$ International Symposium on Wireless Communication Systems, 2011, Aachen, pp.171-175.

[7] M. Shirakabe, A.Morimoto,and N. Miki, “ Performance evaluation of inter-cell interference coordination and cell range expansion in heterogeneous networks for LTE-Advanced downlink" Paper presented at the 8th International Symposium on Wireless Communication Systems (ISWCS), Nov.2011.

[8] 3GPP, TR 36.931, "LTE; Evolved Universal terrestrial Radio Access (EUTRA); Radio Frequency requirements for LTE Pico Node B”, May. 2011.

[9] 3GPP, TS 36.814, "Evolved Universal Terrestrial Radio Access (EUTRA); further advancements for E-UTRA physical layer aspects”, Mar. 2010.

[10] 3GPP, TS 36.423, “X2 application protocol (X2AP),” June. 2011.

[11] D. Lopez-Perez, I. Guvenc, G. de la Roche, M. Kountouris, T. Q. S. Quek, and Z. Jie, "Enhanced intercell interference coordination challenges in heterogeneous networks," IEEE Wireless Communications, vol.18, no.3, June.2011, pp.22-30.

[12] R1-104968, "summary of the Description of Candidate eICIC Solutions,” 3GPP Std., Madrid, Spain, Aug.2010.

[13] 3GPP TS 136.300, "Evolved Universal Terrestrial Radio Access (EUTRA) and Evolved Universal Terrestrial Radio Access Network (EUTRAN); Overall description; Stage 2 (Release 10),” 2011.

[14] K. Okino, T. N., C. Yamazaki, H. Sato, Y. Kusano, "Pico Cell Range Expansion with Interference Mitigation toward LTE-Advanced Heterogeneous Networks,” IEEE International Conference on the In Communications Workshops (ICC), June.2011.

[15] J.Pang, J.Wang, G.Shen, Q.jiang and J.Liu, “Optimized Time-Domain Resource Portioning for Enhanced Intercell Interference Coordination in Heterogeneous Networks," IEEE Wireless Communication and Networking Conference: MAC and Cross-Layer Design, 2012, pp.1613-1617.

[16] L.Jiang and M.Lei, "Resource Allocation for eICIC Scheme in Heterogeneous Networks," IEEE $23^{\text {rd }}$ International Symposium on personal, Indoor and Mobile Radio Communications (PMRC), Sep.2012, pp.464-469.

[17] J. Nash, The Bargaining Problem, Econometrica, vol. 18, no. 2, Apr. 1950, pp. 155-162

[18] Han, Z, Ji, Z and Liu, K J., Fair Multiuser Channel Allocation for OFDMA Networks Using Nash Bargaining Solutions and Coalitions.,2005, IEEE Transactions on Communications, vol. 53, pp. 1366-1376.

[19] M.Al-Rawi, J.Huschke, and M.Sedra, "Dynamic Protected-Subframe Density configuration in LTE Heterogeneous Networks," $21^{\text {th }}$ international Conference on Computer Communication and networks (ICCCN), Aug.2012, pp.1-6.

[20] J.Wang, X.She, and L.Chen, "Enhanced Dynamic Inter-cell Interference Coordination Scheme for LTE-Advanced," IEEE $75^{\text {th }}$ Vehicular Technology Conference (VTC spring), May.2012, pp.1-6.

[21] L.Jiang and M.Lei, "CQI Adjustment for eICIC Scheme in Heterogeneous Networks," IEEE $23^{\text {rd }}$ International Symposium on personal, Indoor and Mobile Radio Communications (PMRC), Sept.2012, pp.487-492. 